This document is published in:

“IEEE Network, January-February 2011, 25 (1), 14-19.

Doi: http://dx.doi.org/10.1109/MNET.2011.5687948."

(C) 2011 IEEE. Personal use of this material is permitted. Permission from IEEE must be obtained for all other uses, in any current or future media, including reprinting/republishing this material for advertising or promotional purposes, creating new collective works, for resale or redistribution to servers or lists, or reuse of any copyrighted component of this work in other works. 


\title{
On Providing Metro Ethernet Services over Transparent WDM Optical Rings
}

\author{
Gerson Rodríguez de los Santos, Manuel Urueña, José Alberto Hernández, and David \\ Larrabeiti, Universidad Carlos III de Madrid
}

\begin{abstract}
The long list of benefits (especially cost and capacity) of Ethernet LANs has made the IEEE, the ITU-T, and the Metro Ethernet Forum define the requirements for taking Ethernet beyond the local area, toward the metropolitan region. In addition, the ever-increasing traffic demands of new applications and users can only be met by the huge bandwidth capacity provided by optical fibers. This work studies how to provide metro Ethernet services over transparent tunable-transmitter fixed-receiver WDM optical ring networks. A new adaptation layer of ME to WDM is proposed, and its benefits and drawbacks are studied. It is shown that such a transparent WDM ring network can be seen as a logical full-mesh topology by the upper ME layer, thus reducing to one the number of optical-electronic-optical conversions per unicast frame. Additionally, two different approaches are proposed in the case of broadcast/multicast traffic, since this may bring scalability difficulties in ring topologies.
\end{abstract}

It is estimated that about 90 percent of total traffic traversing the Internet has been both generated on and destined to Ethernet LANs. Indeed, the success of Ethernet in the local area is mainly justified by its low cost and great capacity features. For these reasons, a number of standardization bodies such as the IEEE, the International Telecommunication Union - Telecommunication Standardization Sector (ITU-T), and the Metro Ethernet Forum (MEF) have put significant effort into devising the requirements to take Ethernet beyond the local area and extend it to the metropolitan region. Such efforts have given rise to the IEEE 802.1ad provider bridges (PB), IEEE 802.1 ah provider backbone bridges (PBB), and IEEE 802.1Qay provider backbone bridges with traffic engineering (PBBTE), the latter completed in 2009.

Besides, the research community has also understood that only optical networks can provide the capacity required to overcome the ever-increasing bandwidth demands of users, especially due to the rise of video streaming applications such as IP television (IPTV) and video on demand (VOD). Indeed, optical fibers provide an amount of bandwidth on the order of terahertz, which, thanks to wavelength-division multiplexing (WDM), can provide a potential capacity of terabits per second.

In addition, the local loop is beginning to migrate to optical-based solutions by means of fiber to the x (FTTx, either home, cabinet, or building), to satisfy the bandwidth demands of the next generation of applications. In light of this, the Ethernet passive optical network (EPON) has been proposed as the Ethernet-based optical access solution to aggregate the traffic generated by thousands of end-subscribers up to the central office, which provides the bridge toward the upper metropolitan area network (MAN). At present, most MAN solutions are based on complex and costly time-division multiplexing (TDM)-based synchronous optical network/digital hierarchy (SONET/SDH) metropolitan networks, which typically provide OC192 circuits operating at $10 \mathrm{~Gb} / \mathrm{sec}$ over an underlying optical ring infrastructure. SONET/SDH networks are limited by the electronic processing bottleneck, and newer all-optical transparent WDM-based networks have already been proposed by the optical research community to replace SONET/SDH networks [1-3].

An easy approach to bringing cost effectiveness to optical metro networks is by using the so-called tunable-transmitter fixed-receiver (TT-FR) optical WDM rings. In these settings, every node in the ring is provided with a fixed receiver tuned on a specific (often dedicated) wavelength for the reception of data, together with a tunable transmitter (or a set of fixed transmitters) used to send data on a particular wavelength. Often, the number of wavelengths equals the number of nodes in the ring, thus allowing each node to have a dedicated home channel for reception. Transmission of data to a given node only requires tuning the transmitter to the destination wavelength, since only such a node is expected to be listening on such a wavelength, while all other nodes transparently bypass the information on all wavelengths except its reception one. A large number of TT-FR-based control mechanisms have been proposed in the last decade to manage access to the (shared) dedicated home channel of a given destination node [2].

Thus, given the fact that the access networks of the future are very likely to be optical, and possibly Ethernet-based (EPON and the emerging WDM-EPON), plus the recent efforts put into extending the Ethernet domain toward the metropolitan area, it seems that deploying metro Ethernet (ME) services over transparent all-optical WDM rings could be a possible scenario for the MAN of the future. This work focuses on studying the requirements and features ME may demand from a transparent TT-FR-based WDM ring network. 


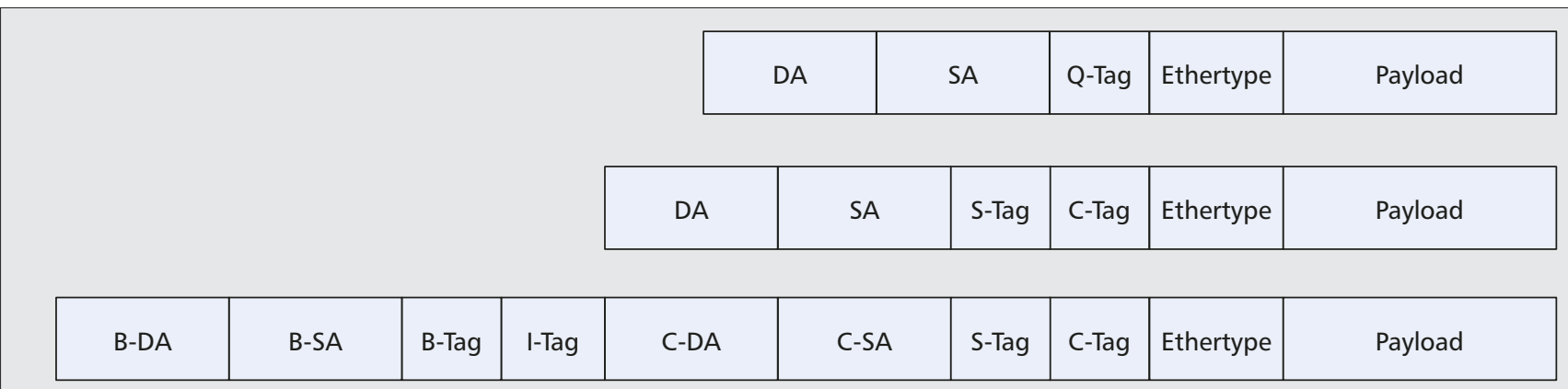

Figure 1. 802.1Q frame (top), 802.1ad frame (middle), and 802.1 ah frame (bottom).

To do so, the rest of this article is organized as follows. The next section briefly revises how ME operates in the MAN using tags to define Service Instances and Virtual LANs and how forwarding is performed. We then briefly introduce optical WDM rings and propose several forwarding and broadcasting efficient mechanisms, as well as a transparent adaptation layer for ME over TT-FR WDM rings. We then illustrate the operational principles of the suggested broadcast/multicast mechanisms and evaluate their performance. Finally, we conclude this work with a summary of its main contributions.

\section{Metro Ethernet Review}

Ethernet poses a number of challenges as a switching technology in large scenarios, for the following reasons [4]:

- Scalability: A conventional MAN may handle thousands of end stations, hence backward learning of the medium access control (MAC) addresses of transit frames could make forwarding tables huge.

- Stability and traffic engineering: The Spanning Tree Protocol (STP) converges very slowly in large scenarios, and often causes forwarding instabilities.

- Availability and resilience: Failure restoration mechanisms are key features required in metro scenarios, in order to be competitive with other MAN technologies such as SONET/SDH.

- Quality of service in the MAN is quite challenging.

Most of these issues have been partially or totally solved by the metro Ethernet standards reviewed next.

\section{Preliminaries: IEEE 802.1Q and 802. lad}

Virtual LANs (VLANs) were originally conceived in IEEE 802.1Q [5] as a means to arrange multiple workstations into customized logical network topologies, as if they all belong to the same physical LAN. Such a logical clustering of stations within the same physical topology also allowed the partitioning of broadcast domains, thus improving the global performance of LANs.

Packets from VLANs must include a four-byte header between the source address and the Ethertype, as noted in Fig. 1. Such an extra header comprises a new Ethertype (of value $0 \times 8100$ ) and a two-byte VLAN tag (the Q-tag), from which 12 bits are used as the VLAN identifier. Twelve-bit VLAN identifiers allow for a maximum of 4094 different VLANs, an upper bound that is clearly insufficient to provide VLAN-based services in a carrier-scale network.

For ME scenarios, the IEEE 802.1ad [6] PB proposed to add an extra 802.1Q tag (referred to as S-tag or service tag) to the previous Q-tag (now renamed C-tag or customer tag) in order to further extend the number of possible VLANs on the same domain (Fig. 1). With this Q-in-Q strategy, a provider may offer service to 4094 customer networks at most, each one with 4094 service VLAN IDs.
Q-in-Q partially solved the limitation of VLAN IDs but brought other drawbacks related to the fact that frames enter the operator's network with the original source and destination MAC addresses [4]. First, there is a potential security risk in having all MAC addresses visible outside the domain of the customer network. Second, frame forwarding based on customer end station MAC addresses require bridges to learn the MAC addresses of all customer networks. Finally, changes triggered by STP at any customer network may affect the operation, administration, and maintenance (OAM) of the whole network.

In summary, subsequent standards focused on separating the STP and the addressing domain of the customers' networks from the operator transport infrastructure, bringing the IEEE 802.1ah standard.

\section{IEEE 802. I ah Provider Backbone Bridges}

IEEE 802.1ah addressed the former problems [7] by defining two types of bridges: backbone edge bridges (BEBs) and backbone core bridges (BCBs). The former type is in charge of encapsulating/decapsulating frames from/to outside of the PBB network (PBBN). Essentially, when an incoming frame from a customer network arrives at the BEB, this includes a new Ethernet header with its own MAC address (as source address) and the address of the egress BEB to which the destination end station is attached, together with two new VLAN IDs, the B- and I-Tags. The 802.1ah header can be seen in Fig. 1, where B-DA and B-SA refer to the backbone destination and source addresses, respectively; that is, the address of the egress and ingress switch in the provider's network (destination and source BEBs), and the B-Tag and I-Tag refer to the backbone and service VLAN IDs, respectively. The B-Tag is a conventional 12-bit VLAN identifier used to provide VLAN clustering and broadcast domain partitioning inside the core of the network. However, the new I-Tag is a 24-bit identifier to define different service instances (up to 16 million) and may be seen as a logical service identifier. The ITags are carried within the B-Tags and are used only at the BEBs to identify service instances $[7,8]$, which can be pointto-point (E-LINE), point-to-multipoint (E-TREE), or multipoint-to-multipoint (E-LAN).

This MAC-in-MAC encapsulation approach brings many benefits [4]. First, the BCBs do not need to learn end-station MAC addresses, but perform the forwarding based on BEB MAC destinations only (together with the B-Tag), thus greatly improving the scalability of metro Ethernet. Therefore, the MAC addresses of end stations are somehow hidden (encapsulated) within the new IEEE 802.1 ah frame and protected from being learned by the BCBs. Second, the STP traffic exchanged among customer sites is also encapsulated; thus, it never affects the provider's network. Finally, the operator can keep its own network under control by managing the paths and mechanisms concerning the forwarding between two BEBs, thanks to the use of the B- and I-Tags [9]. 


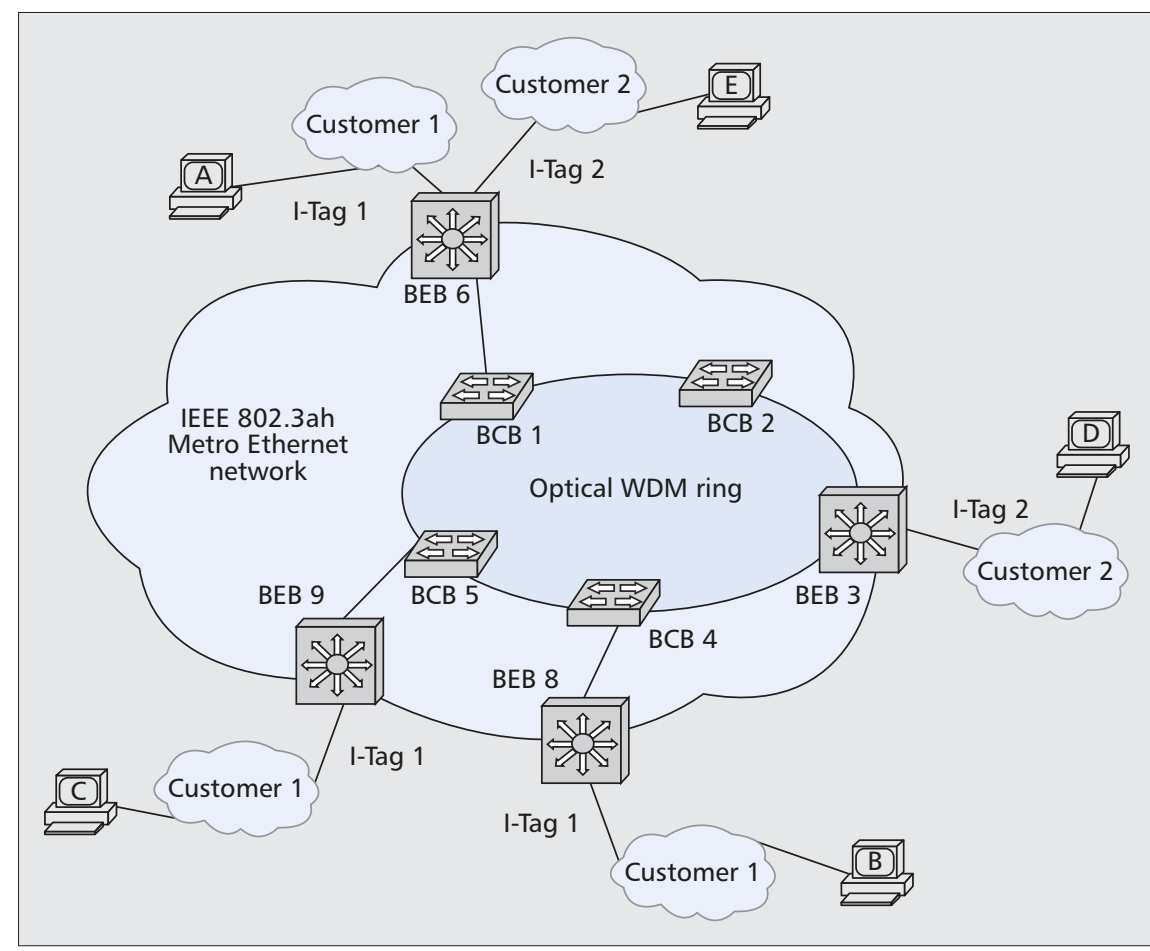

Figure 2. Reference scenario.
Concerning broadcast, the easiest implementation considers the replication of the broadcast frame over all destination home channels. However, this strategy is clearly bandwidth consuming, especially when the number of nodes in the ring is large.

Alternatively, frame broadcast can be implemented by sending a single frame hop-by-hop along the ring following a store-and-forward basis. This strategy scales with the number of nodes in terms of bandwidth consumption, but has two main drawbacks:

-The frames must suffer optical-electronic-optical (OEO) on each node, thus increasing latency (and removing transparency in broadcast transmission).

- Some extra control is necessary: the source node must remove its broadcast frame after a loop around the ring.

The next section studies how to adapt metro Ethernet to TT-FR WDM rings.
In summary, thanks to the MAC-in-MAC strategy, the core of a metro Ethernet network (i.e., the BCBs) can easily be operated, administrated, and managed, and may simply comprise a network of conventional (high-performance) Ethernet switches, since their forwarding is based on the BDA and BTag only.

Combining the simplicity of ME forwarding with the highcapacity and transparency features of TT-FR WDM networks may be the choice for next-generation provider network MANs. The next sections briefly review TT-FR WDM optical rings and how to combine both worlds into a high-performance cost-effective ME over WDM MAN.

\section{A Review of Transparent TT-FR WDM Optical Rings}

In TT-FR WDM ring topologies with $N$ nodes and $W$ wavelengths, each node in the ring has got a tunable laser (tunable to any wavelength) and a receiver, which is always fixed to the same reception wavelength. In the cases where $N=W$, each node in the ring can be provided with its own reception wavelength (often called dedicated home channel), and at the same time, the tunable laser provides each node with a means to communicate (directly) with all others, just by tuning its laser to the appropriate reception wavelength of the destination node. Transparency is achieved because all nodes bypass all wavelengths except their dedicated home channel, which is stripped off the ring by its owner. Thanks to WDM and transparency, each node is provided with a logical unidirectional channel to all other nodes in terms of transmission, but it can only receive frames from a single wavelength.

This architecture has been widely studied in the literature because of its simplicity, and a number of MAC protocols have been defined to arbitrate channel access, essentially based on multitoken passing (MTIT), wavelength inspection (DBORN), timeslot reservation (MAWSON, RingO), or a dedicated control channel (HORNET). Further details can be found at [2, Ch. 13].

\section{Metro Ethernet over WDM Rings}

Figure 2 shows a reference scenario to analyze ME over WDM optical rings. In this scenario, three end-stations from Customer Network no. 1 are connected to an IEEE 802.1ah network comprised by four BEBs (each of them interface a given customer network) and four BCBs. As shown, the four BCBs (nodes 1, 2, 4, and 5) together with BEB number 3 are aligned along a ring topology. Assuming that the nodes in the ring are connected with optical fibers, it is possible to build a transparent TT-FR-based optical ring to exploit the benefits of WDM and transparency. To do so, it is first necessary to define a convergence layer that adapts the features of metro Ethernet to the underlying WDM ring network.

We consider the transmission example of a frame sourced at station A from customer network 1 and destined to station B. Figure 3 shows the structure of this frame of reference before and after entering the metro Ethernet network at node BEB 6. Remark that this frame must traverse nodes 6-1-2-3 4-8 to reach its destination.

If the nodes in the ring are just conventional ME nodes, the reference frame will suffer OE conversion, processing, and EO conversion on every node in the ring in order to be forwarded to the next one. In this light, the ring is opaque and acts as a store-and-forward network, and consequently does not exploit the benefits of WDM and transparency of optical rings. The next section examines two different ways to provide transparency and exploit the bandwidth provided by WDM: the logical full mesh topology over TT-FR and the logical full mesh topology over TT-FR with hop-by-hop broadcast.

\section{First Approach: Logical Full Mesh over TT-FR}

This case considers a TT-FR WDM ring topology (BBs 1 to 5 in Fig. 2), where every node in the ring is provided with a dedicated home channel for reception (for notation simplicity, node $i$ listens on wavelength $\lambda_{i}$ ). Essentially, intermediate nodes have the ability to optically bypass traffic from all channels (wavelengths) except its dedicated one; thus, it always removes all the packets from its reception home channel. In light of this, the reference frame shown in Fig. 3 enters the 


\begin{tabular}{|l}
\hline \begin{tabular}{|l|l|l|l|l|l|l|l|l|l|l|l|l|l|}
\hline MAC 8 & MAC 6 & B-Tag & I-Tag & MAC B & MAC A & C-Tag & Ethertype & PAC A & C-Tag & Ethertype & Payload \\
\hline Optical node 4 & Optical node 1 & MAC 8 & MAC 6 & B-Tag & I-Tag & MAC B & MAC A & C-Tag & Ethertype & Payload \\
\hline
\end{tabular}
\end{tabular}

Figure 3. Reference frame: Before entering the ME network (top), after MAC-in-MAC encapsulation (middle), and after entering the optical ring (bottom).

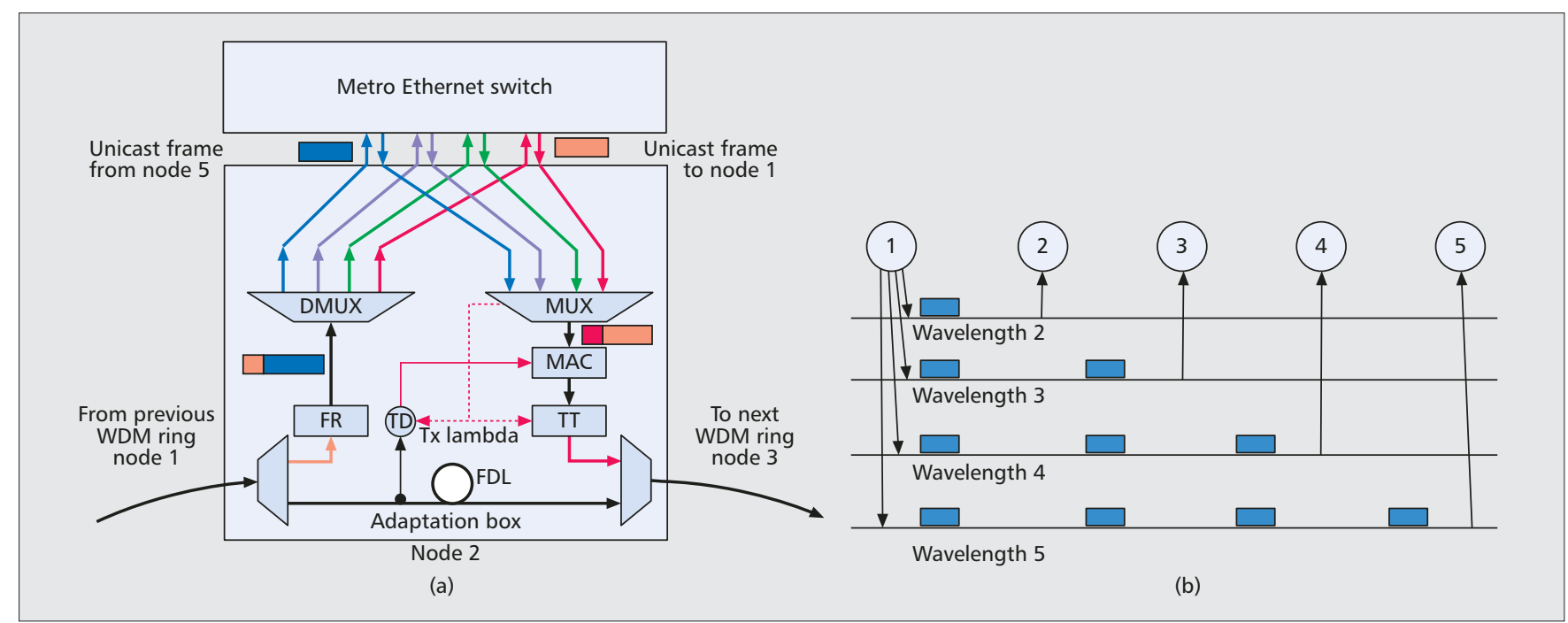

Figure 4. a) Architecture for an ME TT-FR node; b) broadcasting of frames on a full mesh TT-FR WDM ring.

ME network at BEB 6 and must ideally traverse BCBs 1-2-3-4 to arrive at BEB 8, where the frame egresses the ME network toward its destination, station $\mathrm{B}$. The idea is to exploit the potential of WDM and transmit this frame transparently around the optical ring, that is, bypassing (all optically) nodes 2 and 3 . To do so, node BCB 1 must take the following steps upon reception of our frame of reference: examine the destination address (this is BEB 8) and understand that the next ME hop is $\mathrm{BCB} 4$; and consequently forward the frame on BCB 4's home wavelength (i.e., $\lambda_{4}$ ).

To accomplish this, we propose the architecture of Fig. 4a, which combines a conventional metro Ethernet switch with four in/out ports together with an adaptation box to the TTFR ring. Four in/out ports interfacing the ME box are necessary to hide the underlying TT-FR network from the upper ME switch. The idea is to make the Ethernet switch think that it is directly connected to the other four nodes in the ring as in a full mesh topology. To do so, it is ideally required that the ME switch sends and receives packets from node $i$ on the $i$ th port in order to apply backward MAC learning. It is the role of the adaptation box to translate this behavior into the proper operation mode of the TT-FR ring.

Basically, when the adaptation box receives a packet from the upper ME switch on input port $i$, this packet is intended for the $i$ th switch in the ring and must be optically transmitted on the home channel of the $i$ th node, $\lambda_{i}$. Hence, concerning transmission, the adaptation box is in charge of mapping the upper ports to the appropriate wavelengths (frames from port $i$ must go to wavelength $\lambda_{i}$ ). This way, all-optical transmission transparency is achieved in the WDM ring.

As previously mentioned, there are multiple proposals in the literature to arbitrate the access to a TT-FR ring in order to avoid collisions. In this case we consider a simple MAC layer that employs a tunable photo-detector (TD) and a fiber delay loop (FDL) following the DBORN architecture [3]. When the station needs to send a frame to a station with home channel $\lambda_{i}$, it first checks whether the TD has detected any incoming frame in this wavelength. If not, it may start transmitting its own frame since, even if some frame arrives just after the check, the FDL will delay it enough time to avoid a collision.

Concerning reception, the operation of the adaptation box must be just the opposite, that is, mapping the incoming optical frames from the ring to the appropriate port of the ME switch. In other words, frames coming from node $i$ must go to the $i$ th port. However, a TT-FR node has no means to distinguish the source node of a given frame received, since all of them arrive on the same wavelength: the node's dedicated home channel. This issue can be solved by the adaptation box by adding an extra optical-ring related header that states which node generated each frame, as well as the destination node. Therefore, every frame entering the optical ring must include an identifier of both the destination and source nodes. This ring adaptation header must be added/removed by the adaptation box to the upper ME switch. Then, if the adaptation box is capable of forwarding frames between wavelengths and ME ports appropriately, the ME switch will have a view of the ring as a full mesh topology and backward MAC learning can operate adequately, as in conventional Ethernet.

Finally, when the ME switch sends a broadcast frame on all its output ports, this frame is then replicated on all the wavelengths of the ring by the adaptation box with the appropriate 


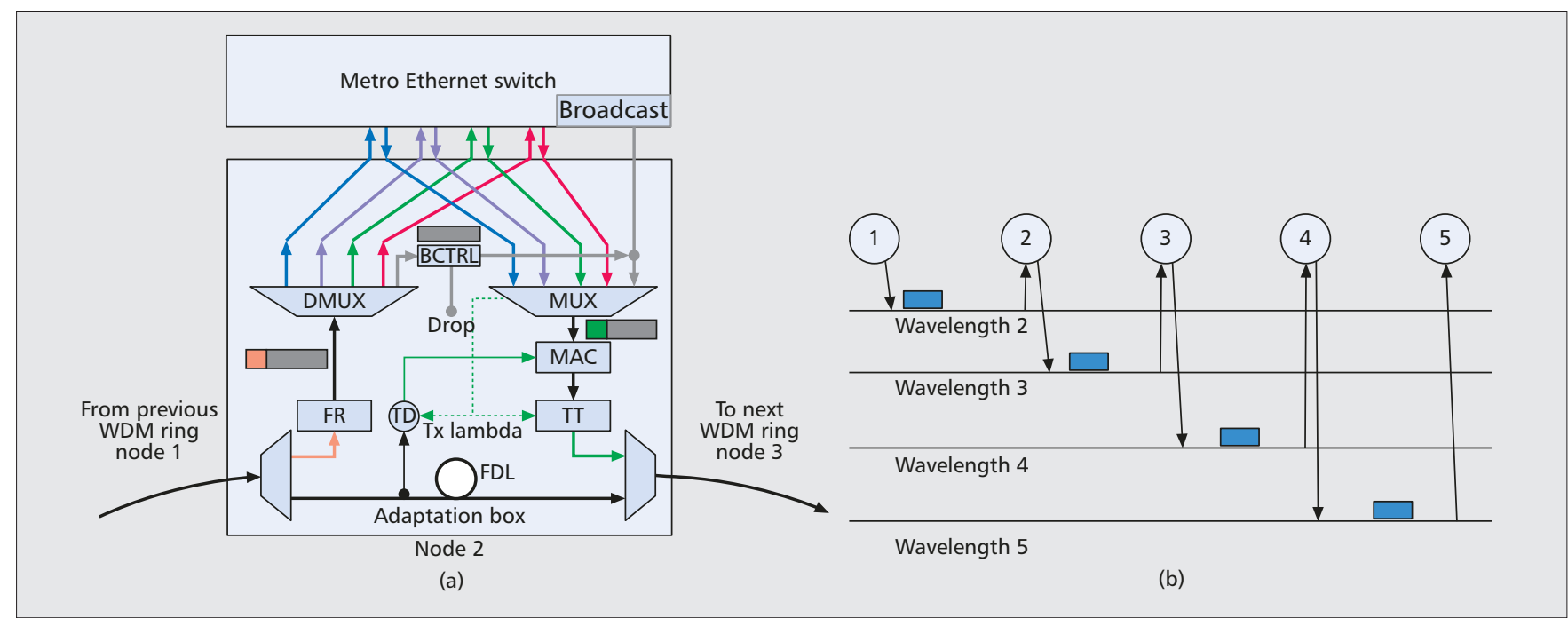

Figure 5. a) Architecture for an ME TT-FR node with hop-by-hop broadcast; b) broadcasting of frames on a full mesh TT-FR WDM ring with hop-by-hop broadcast.

ring adaptation header: broadcast identifier and source node identifier. Remark that such broadcasting may occur for two reasons in Ethernet:

- The frame has a multicast or broadcast address (e.g., Address Resolution Protocol [ARP] queries, IPTV services).

- The MAC destination address is unknown to the switch, and must therefore forward the frame to all output ports.

This strategy is very simple and quite easy to implement, but clearly imposes a capacity penalty since the same frame must traverse the same links on different wavelengths (Fig. $4 \mathrm{~b})$. The next section proposes an alternative mechanism to reduce such bandwidth consumption.

\section{Second Approach: Logical Full Mesh with Hop-by-Hop Broadcast}

This approach combines the benefits of a TT-FR ring concerning optical transparency with the hop by-hop transmission of broadcast/multicast traffic. Concerning unicast transmission, the adaptation box operates exactly the same as in the previous case, mapping ME ports to wavelengths and including an extra adaptation header to enable one-to-many reception mapping. The difference with the previous case lies in the transmission of broadcast/multicast/unknown frames, which are transmitted hop by hop along the ring in order to avoid duplicated frames on all wavelengths (Fig. 5b).

However, since now the broadcast frames must be handled by the adaptation box (Fig. 5a) instead of the ME switch, it is necessary that the switch sends all broadcast/multicast or frames with unknown destinations by a specific port to the adaptation box instead of duplicating the broadcast frame by all its output ports. Also, the adaptation box needs some additional logic that tells every node whether to forward broadcast frames to the next node in the ring or strip the frame off the ring. The easiest strategy is to employ source stripping; that is, the source node must strip the frame off the ring once it has looped the ring.

Two additional improvements can be made concerning the hop-by-hop broadcasting of frames with unknown destination:

- If a given node acknowledges that this frame is intended for itself, it may strip the frame off the ring rather than circulating it along the ring.

- If a given node acknowledges that this frame is intended for some other node in the ring, it may sent it through the dedicated home channel of such destination node (i.e., transparently rather than hop by hop).

Finally, it is worth noting that hop-by-hop broadcasting requires all frames to suffer OEO conversion on each node, as it operates in a store-and-forward manner. Additionally, the adaptation box is required to keep internally a sorted list of the next nodes in the ring for every B-VLAN, since it must know the next hop for every broadcast frame.

\section{Evaluation}

Table 1 compares the two ME solutions over transparent WDM rings with conventional ME over opaque WDM rings in terms of average bandwidth-link consumption and number of OEO conversions per frame, for both unicast and broadcast frames.

Clearly, the average number of links a unicast frame traverses in an $N$-node ring is $N / 2$, and so is the number of OEO conversions in an opaque ring. Concerning broadcast, since

\begin{tabular}{|llll|}
\hline Method: case & $\begin{array}{l}\text { Conventional } \\
\text { ME opaque ring }\end{array}$ & $\begin{array}{l}\text { Logical full } \\
\text { mesh over TT-FR }\end{array}$ & $\begin{array}{l}\text { Logical full mesh over TT-FR } \\
\text { with hop-by-hop broadcast }\end{array}$ \\
\hline $\begin{array}{l}\text { Unicast: bandwidth consumption } \\
\text { Unicast: OEO conversions }\end{array}$ & $N / 2$ & $N / 2$ & $N / 2$ \\
\hline Broadcast: bandwidth consumption & $N-1$ & 1 & 1 \\
\hline Broadcast: OEO conversions & $N-1$ & $(N-1) N / 2$ & $N-1$ \\
\hline
\end{tabular}

Table 1. Performance evaluation of ME over TT-FR topologies. 
the frame must be received on every node of the ring, $N-1$ OEO conversions are necessary. If a transparent solution is employed (either with or without hop-by-hop broadcast), the number of links on average traversed by a unicast frame is still $N / 2$ but the number of OEO conversions reduces to 1 , since the frame traverses the ring all-optically toward its destination. However, the full mesh topology replicates broadcast frames over all destination wavelengths, which requires an amount of $(N-1) N / 2$ links on average (Fig. 4b) and $N-1$ OEO conversions (one per destination node). The full mesh with hop-by-hop broadcast approach reduces bandwidth consumption to $N-1$, as noted from Fig. $5 \mathrm{~b}$.

Two important conclusions arise from Table 1. First, the use of a transparent TT-FR ring reduces the number of OEO conversions per unicast frame to one conversion only. However, the goal of keeping transparent transmission makes frame broadcasting explode for large $N$ in the first approach. To alleviate this, the hop-by-hop broadcasting of frames reduces bandwidth consumption, but requires OEO conversion and processing on each intermediate node. Hence, choosing one strategy or the other depends on the amount of broadcast traffic expected in the network, the number of nodes in the ring, and the traffic load.

\section{Conclusions}

This article has reviewed current metro Ethernet standards and proposed an adaptation layer to deploy ME over transparent WDM optical rings. Basically, such an adaptation layer requires to map ME switch ports to the appropriate wavelengths of a TT-FR optical ring concerning transmission, and a new adaptation header to differentiate between source nodes concerning reception. Two solutions have been proposed concerning frame broadcast. The first approach requires no changes on the $\mathrm{ME}$ switch but lacks scalability in broadcast traffic delivery. The second one requires additional coordination between the ME switch and the adaptation box to perform store-and-forward transmission of broadcast frames in an ordered manner along the ring. Nevertheless, the use of TTFR-based WDM optical rings results in an all-optical virtual full mesh topology for unicast frames, since all nodes in the ring are just one hop distant thanks to its optical transparent nature.

\section{Acknowledgments}

The work described in this article was carried out with the support of the Building the Future Optical Network in Europe (BONE) project, a Network of Excellence funded by the European Commission through the 7th ICT-Framework Programme. Additionally, the authors are thankful for the support of the T2C2 (grant TIN2008-06739C04-01) and MEDIANET (grant S2009/TIC-1468) projects for the development of this work.

\section{References}

[1] I. M. White et al.., "A Summary of the HORNET Project: A Next-Generation Metropolitan Area Network," IEEE JSAC, vol. 21, no. 9, Nov. 2003, pp. 1478-94.

[2] M. Maier, Optical Switching Networks, Cambridge University Press, 2008

[3] N. Bouabdallah and H. Perros, "Cost-Effective Single-Hub WDM Ring Networks: A Proposal and Analysis," Comp. Net., vol. 51, no. 13, Sept. 2007, pp. 3878-3901.

[4] R. Sanchez, L. Raptis, and K. Vaxevanakis, "Ethernet as a Carrier Grade Technology: Developments and Innovations," IEEE Commun. Mag., vol. 46, no. 9, Sept. 2008 , pp. $88-94$

[5] IEEE Std. 802.1Q-2005, "IEEE Standard for Local and Metropolitan Area Networks: Virtual Bridged Local Area Networks," Dec. 2005.

[6] IEEE Std. 802.1 ad, "IEEE Standard for Local and Metropolitan Area Networks: Virtual Bridged Local Area Networks. Amendment 4: Provider Bridges," May 2006

[7] P. Bottorff and P. Saltsidis, "Scaling Provider Ethernet," IEEE Commun. Mag., vol. 46, no. 9, Sept. 2008, pp. 96-103.

[8] IEEE Std. 802.1 ah, "IEEE Standard for Local and Metropolitan Area Networks: Virtual Bridged Local Area Networks. Amendment 7: Provider Backbone Bridges," Aug. 2008

[9] IEEE Std. 802.1Qay, "IEEE Standard for Local and Metropolitan Area Networks: Virtual Bridged Local Area Networks. Amendment 10: Provider Backbone Bridge Traffic Engineering," Aug. 2009.

\section{Additional Reading}

[1] M. Ali, G. Chiruvolu, and A. Ge, "Traffic Engineering in Metro Ethernet," IEEE Network, vol. 19, no. 2, Mar./Apr. 2005, pp. 10-17.

\section{Biographies}

GERSON RODRIGUEZ DE LOS SANTOS (gsantos@it.uc3m.es) received his Telecommunications Engineering degree in April 2008 and his M.Sc. in telematics engineering in July 2010 at Universidad Carlos III de Madrid, Spain, where he is also pursuing a Ph.D. degree in telematic engineering. $\mathrm{He}$ is currently working at that university as a research assistant on both national and European research projects. His research interests cover the fields of optical transparent networks, network hardware development, and switching in general.

MANUEL URUEÑA received his M.Sc. degree in computer science from Universidad Politécnica de Madrid, Spain, in 2001 and his Ph.D. degree in telecommunications from Universidad Carlos III de Madrid in 2005. At present, he is an assistant professor in telematics engineering at Universidad Carlos III de Madrid. His current research interests range from P2P systems to optical networks. He has been involved in several international and national research projects related to these topics.

JOSÉ ALBERTO HERNANDEZ completed a five-year degree in telecommunications engineering at Universidad Carlos III de Madrid in 2002, and a Ph.D. degree in computer science at Loughborough University, Leicester, United Kingdom, in 2005. He is currently an associate professor at Universidad Carlos III de Madrid. $\mathrm{He}$ has published more than 40 articles in both journals and conference proceedings. His research interests include the areas at which mathematical modeling and computer networks overlap.

DAVID LARRABEITI is professor of switching and networking architectures at Universidad Carlos III of Madrid. Since 1990 he has participated in EU-funded research projects related to next-generation networks and protocols. In 2009-2010 he was visiting researcher at Stanford University under Spanish mobility grant PR2009-0221. He is UC3M's principal investigator at the BONE network of excellence on optical networking. His current research interests include the design of hybrid electro-optical packet switches and multipoint optical communications. 